\title{
NUMERICAL SIMULATION OF HEAT-LOSS COMPENSATED CALORIMETER
}

\author{
YONGSOO CHOI ${ }^{1}$, KOOK JIN JEON $^{2}$, YOUNGHO PARK ${ }^{3} \&$ SANGIL HYUN $^{3}$ \\ ${ }^{1}$ School of Liberal Arts and Basic Science, Hankyong National University, Korea. \\ ${ }^{2}$ Department of Accelerator Science, Korea University Sejong Campus, Korea. \\ ${ }^{3}$ Korea Institute of Ceramic Engineering and Technology, Korea.
}

\begin{abstract}
An analytical model using finite element approximation was applied to determine the amount of heat dissipated in a three-body graphite calorimeter used in the field of dosimetry. The temperature drifts and the heat dissipation of the calorimeter bodies via conduction and radiative transfer during electrical heating were considered to enhance heat insulation for the accurate measurement of absorbed dose. A simulation was applied to the heating and cooling process for both electrical calibration and irradiation. The heat transfer in the calorimeter bodies and wire could be first estimated quantitatively. The radiation energy absorbed into the core during irradiation was estimated in a heat-loss-compensated mode of operation, and the effects of the wire conduction of the thermistor on the radiant heat loss were investigated.

Keywords: Electrical heating, finite element method, graphite calorimeter, heat transfer, radiation energy.
\end{abstract}

\section{INTRODUCTION}

Absorbed dose is a physical quantity that measures mean energy imparted to a unit of mass in medium by ionizing radiation. The absorbed dose to water, which is a substitute material of human body, is of special interest in radiotherapy and radiosurgery where the delivery of the absorbed dose prescribed by radiation treatment planning is critical for the effective treatment. While a calorimeter is known to be the most reliable apparatus for the accurate measurement of the absorbed dose, the main task in the calorimetric measurement is to determine the heat absorbed by an absorber of interest as accurately as possible. A typical graphite calorimeter is three-body graphite calorimeter [1]-[7], which consists of a central core and a surrounding jacket and shield. These three bodies are thermally isolated from each other by air gaps and plastic supports and contain thermistors, which are used for temperature measurements.

During irradiation, where all three bodies are heated uniformly, the temperatures of the bodies increase nearly equally, and no serious temperature difference arises between the core and the surrounding jacket and shield. Therefore, the heat loss from the core is negligible, and the heat absorbed by the core can be determined by standard measurements [1].

In electrical heating where an exact amount of heat is delivered only to the core, most of the energy is retained in the core, causing the temperature of the core to increase. However, some energy is dissipated from the core as heat leakage, which increases the temperature of the surrounding jacket and shield. The heat leakage from the core includes radiative transfer, conduction through air and heat flow through the measurement wires. The heat loss caused by conduction can be minimized by pumping as much air as possible out of the gaps of the calorimeter. The radiative transfer can be reduced by gluing a low emissivity material such as an aluminized Mylar film [8] onto the inner surface of the surrounding jacket and shield. However, the heat flowing out of the core through the measurement wires is very difficult to measure, which might be nontrivial. 


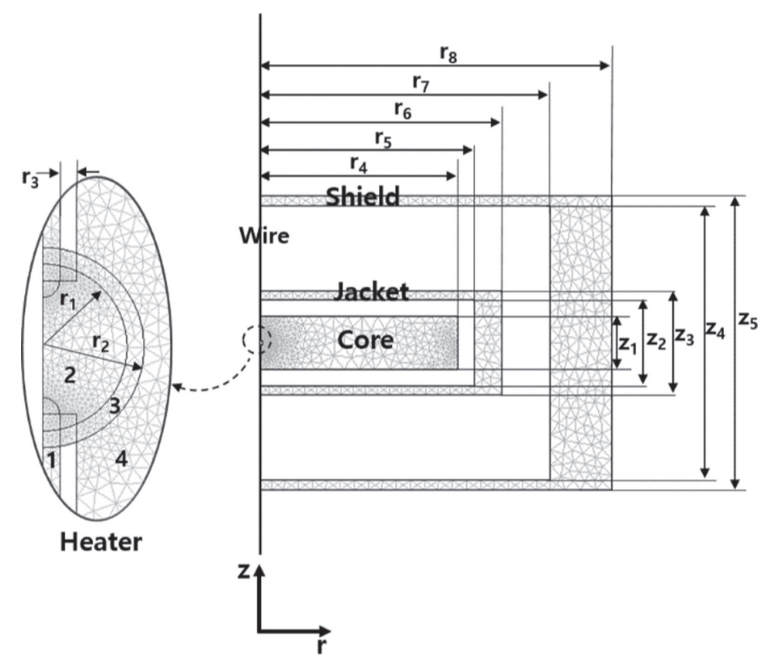

Figure 1: Three-body graphite calorimeter model consisting of the central core and the surrounding jacket and shield. Details regarding the dimensions are shown in Table 1 . The enlarged image shows the heater with number labels; 1 : copper wire, 2: germanium resistor, 3: glass coating, and 4: graphite core.

For the experimental estimation of the amount of heat absorbed by the core, several modes of operation, such as heat-loss compensated [1], adiabatic [2], constant-temperature [5], and quasi-isothermal [6] modes, have been developed, but not many theoretical works [1]-[4] have been performed to clarify the heat-transfer phenomena in the core.

The purpose of this study was to develop the heat-transfer model according to a heat-loss compensated mode of the three-body graphite calorimeter designed by Domen and Lamperti [1] by modeling the heat transport in the calorimeter bodies using the finite element method. This method is very useful because it can describe the heat transfer in the calorimeter bodies both qualitatively and quantitatively. The model was applied to a simplified calorimeter designed to have axial symmetry, as shown in Fig. 1. For the calculation of the realistic dimensions and the characteristics of the calorimeter bodies, a thermistor heater and copper wires were used. A qualitative comparison of the temperature behavior of the three-body calorimeter for the several hundreds of seconds of heating and cooling between the simulation and previously reported values [1] was performed. A quantitative description of the amount of heat transfer in the three-body calorimeter for the electrical calibration and irradiation is also provided. The radiation energy absorbed into the core during irradiation was estimated using the heat-loss compensated calorimeter model. On the basis of the results, the effects of the wire conduction and the positions of the thermistor on the heat loss were investigated.

\section{SIMULATION METHODS}

\subsection{Calorimeter structure and simulation model}

A three-body graphite calorimeter with two-dimensional axial symmetry was modeled using a finite-element method of COMSOL Multiphysics [9], which is a commercial 
Software based on the finite element method for simulating multi-physics phenomena. As shown in Fig. 1, the model consists of three parts: the core, the jacket and the shield. The core and jacket have the same volume. All the parts are made of graphite. For electrical calibration runs, the core contains a heater connected to a 20-mm-long copper wire. The heater at the center of the model is coated with glass for electrical insulation. Details regarding the dimensions and materials of the calorimeter bodies, the heater, and the wires connected to the heater in the simulation model are shown in Table 1. The characteristics of the materials involved in the model are presented in Table 2.

Thermistors for measurements of the temperature in each body of the calorimeter are not included in the model because the two-dimensional axial symmetry model does not allow a point-typed thermistor in each body. The gaps between the bodies containing air at a pressure of less than $10^{-4}$ Torr are excluded from the model for simplicity. The heat flow between the bodies is allowed only through radiation transfer, neglecting conductive and convective heat transfer.

The heat flow and distribution due to the electrical heating were solved using the AC/DC and Heat Transfer modules of COMSOL Multiphysics. Initially, all the materials were at the same temperature, which is typically room temperature. An electric current can be supplied to the heater by applying an electric voltage to the ends of wire. Figures 2(a) and (b) show plots of the electric potential distribution and electric current density near the heater, where the red and blue colors represent high and low values, respectively. The amount of resistive heat in the heater is calculated using the Conductive Media DC application mode in the AC/ DC module [10]-[12] according to the equation,

$$
-\nabla \cdot\left(\sigma \nabla V-J^{\mathrm{e}}\right)=Q_{\mathrm{j}},
$$

where $\sigma, V, J^{\mathrm{e}}$, and $Q_{\mathrm{j}}$, are the electric conductivity, the voltage, the external current source, and the heat source $\left(\mathrm{W} / \mathrm{m}^{3}\right)$, respectively. Figure $2(\mathrm{c})$ shows a plot of the distribution of the

Table 1: Dimensions and types of materials of the calorimeter bodies, heater and wires in the simulation model.

\begin{tabular}{lccc}
\hline Components & Symbol & Dimensions $(\mathbf{m m})$ & Material \\
\hline Heater & $r_{1}$ & 0.125 & $\mathrm{Ge}$ \\
Coating & $r_{2}$ & 0.150 & Glass \\
Wire & $r_{3}$ & 0.025 & $\mathrm{Cu}$ \\
Core & $r_{4}$ & 10.100 & Graphite \\
& $z_{1}$ & 2.740 & \\
Jacket & $r_{5}$ & 11.000 & Graphite \\
& $r_{6}$ & 12.400 & \\
& $z_{2}$ & 4.450 & \\
& $z_{3}$ & 5.240 & Graphite \\
Shield & $r_{7}$ & 14.900 & \\
& $r_{8}$ & 17.995 & \\
& $z_{4}$ & 13.980 & \\
& $z_{5}$ & 15.020 & \\
\hline
\end{tabular}


Table 2: Characteristics of the materials used in the model.

\begin{tabular}{lccccc}
\hline Materials & $\begin{array}{c}\text { Electric } \\
\text { resistivity } \\
(\boldsymbol{\Omega} \cdot \mathbf{m})\end{array}$ & $\begin{array}{c}\text { Temperature } \\
\text { coefficient }(\mathbf{1} / \mathbf{K})\end{array}$ & $\begin{array}{c}\text { Thermal } \\
\text { conductivity } \\
(\mathbf{W} / \mathbf{m} \cdot \mathbf{K})\end{array}$ & $\begin{array}{c}\text { Heat } \\
\mathbf{D e n s i t y} \\
\left.\mathbf{( k g} / \mathbf{m}^{\mathbf{3}}\right)\end{array}$ & $\begin{array}{c}\text { Capacity } \\
(\mathbf{J} / \mathbf{k g} \cdot \mathbf{K})\end{array}$ \\
\hline $\mathrm{Ge}$ & $4.7 \times 10^{-1}$ & -0.034 & 60 & 5327 & 310 \\
$\mathrm{Glass}$ & $1.0 \times 10^{9}$ & $0.55 \times 10^{-6}$ & 1.38 & 2203 & 703 \\
$\mathrm{Cu}$ & $1.72 \times 10^{-8}$ & $3.96 \times 10^{-3}$ & 390 & 8930 & 390 \\
Graphite & $1.17 \times 10^{-5}$ & $2.0 \times 10^{-6}$ & 115 & 1760 & 712 \\
\hline
\end{tabular}
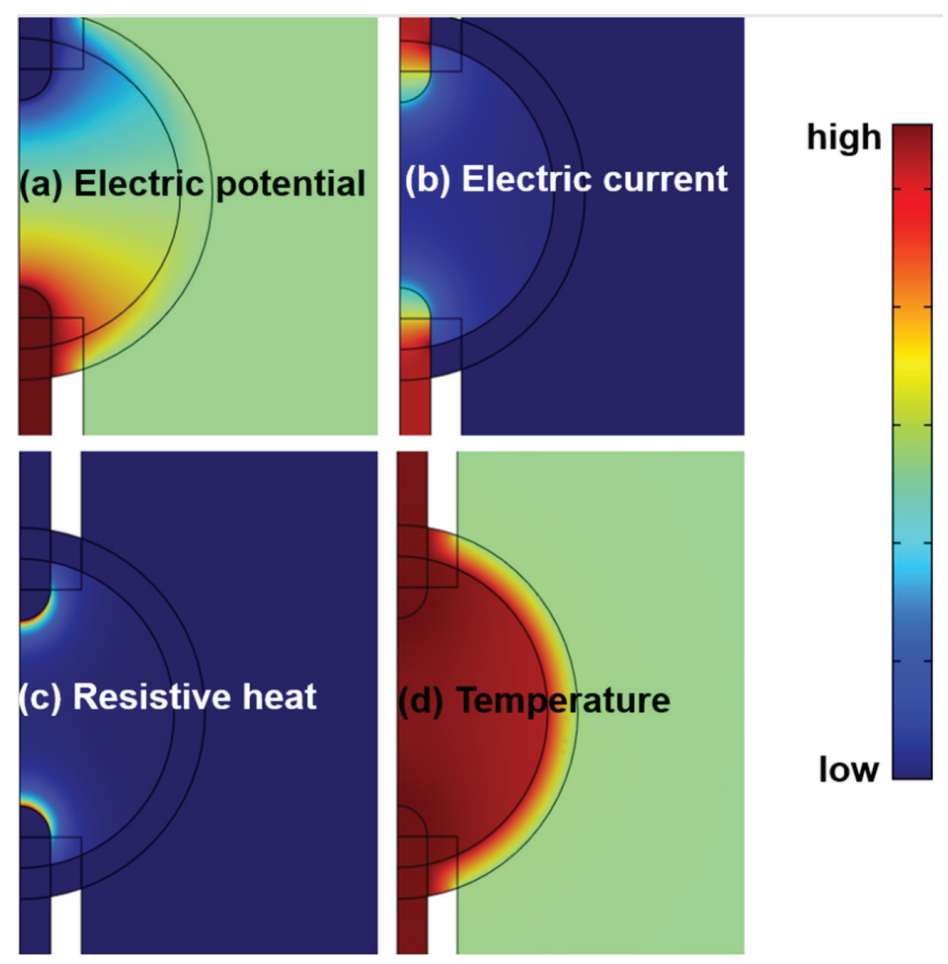

Figure 2: (a), (b), (c), and (d) show plots of the electric potential, electric current density, resistive heat, and temperature distributions, respectively, near the heater during the electrical heating process. The gradient from red to blue represents high values to low ones.

energy due to resistive heat near the heater, where red and blue colors represent high and low values, respectively. The electric conductivity varies with respect to the temperature, as follows:

$$
\sigma=\sigma_{0}\left(1+\alpha\left(T-T_{\text {ref }}\right)\right)
$$


where $\sigma_{0}, \alpha, T$, and $T_{\text {ref }}$ are the electric conductivity at the reference temperature, the temperature coefficient, the temperature, and the reference temperature, respectively. The temperature distribution was obtained using the General Heat Transfer application mode in the Heat Transfer module [13], [14] according to the following equations:

$$
\begin{gathered}
\delta_{\mathrm{ts}} \rho C_{\mathrm{p}} \frac{\partial T}{\partial t}+\nabla \cdot(-k \nabla T)=Q, \\
-\mathbf{n} \cdot(-k \nabla T)=h\left(T_{\mathrm{inf}}-T\right)+\frac{\varepsilon}{1-\varepsilon}\left(J_{0}-s T^{4}\right)+Q,
\end{gathered}
$$

where $\delta_{\mathrm{ts}}$ is a time-scaling coefficient, $\rho$ is the density, $C_{\mathrm{p}}$ is the heat capacity $(\mathrm{J} / \mathrm{kg} \cdot \mathrm{K}), k$ is the thermal conductivity $(\mathrm{W} / \mathrm{m} \cdot \mathrm{K}), Q$ is the volume heat source $\left(\mathrm{W} / \mathrm{m}^{3}\right), \mathbf{n}$ is the surface normal vector, $h$ is the convective heat transfer film coefficient $\left(\mathrm{W} / \mathrm{m}^{2} \mathrm{~K}\right), T_{\mathrm{inf}}$ is the temperature of the convection coolant, $\varepsilon$ is the surface emissivity, $J_{0}$ is the surface radiosity expression $\left(\mathrm{W} / \mathrm{m}^{2}\right)$, and $s$ is the Stefan-Boltzmann constant. Figure 2(d) shows a plot of the temperature distribution near the heater, where the red and green areas represent high and low values, respectively. At the emitter boundary, the model considers the radiation (surface-to-surface) in the boundary condition. It simulates the mirrors by considering the radiation on the inner boundaries of the jacket and shield and applying a low emissivity of 0.05. This models the Mylar coating film on the boundaries, which is used for reducing the heat that flows out of the core and jacket. The outer boundaries of the core and jacket have the emissivity of graphite, i.e. 0.7. The outer boundary of the shield has the emissivity of graphite but is at the ambient room temperature. The ends of the wire have the boundary condition of a fixed room temperature, and the other boundaries have an emissivity of 0.05 .

\subsection{Heat-loss compensated model}

As Domen et al. [1] suggested, the heat-loss compensated calorimeter model assumes that each of the three calorimeter bodies is free of a temperature gradient and that all the heattransfer coefficients and heat capacities are constant. The temperature change of each body with time $t$ is governed by the following differential equations in matrix form:

$$
\begin{gathered}
\mathbf{C} \frac{d \boldsymbol{T}}{d t}=\boldsymbol{P}-\boldsymbol{K} \boldsymbol{T}, \\
\boldsymbol{C}=\left(\begin{array}{l}
C_{1} \\
C_{2} \\
C_{3}
\end{array}\right), \quad \boldsymbol{T}=\left(\begin{array}{l}
T_{1} \\
T_{2} \\
T_{3}
\end{array}\right), \quad \frac{d \boldsymbol{T}}{d t}=\left(\begin{array}{c}
\frac{d T_{1}}{d t} \\
\frac{d T_{2}}{d t} \\
\frac{d T_{3}}{d t}
\end{array}\right), \quad \boldsymbol{P}=\left(\begin{array}{l}
P_{1} \\
P_{2} \\
P_{3}
\end{array}\right)
\end{gathered}
$$

and 


$$
\boldsymbol{K}=\left(\begin{array}{ccc}
K_{1} & -K_{1} & 0 \\
-K_{1} & K_{1}+K_{2} & -K_{2} \\
0 & -K_{2} & K_{2}+K_{3}
\end{array}\right),
$$

where $T_{i}, C_{i}, P_{i}$, and $K_{i}$ represent the temperature increase of the bodies above the initial equilibrium temperature, the heat capacity of the bodies, the power provided to the bodies, and the heat-transfer coefficient of the bodies, respectively. The lower indices $i=1,2,3$ represent the core, jacket, and shield body of the calorimeter, respectively. The asymptotic temperature increments are:

$$
\begin{gathered}
T_{1}(\infty)=\frac{P_{1}}{K_{1}}+\frac{P_{1}+P_{2}}{K_{2}}+\frac{P_{1}+P_{2}+P_{3}}{K_{3}} \\
T_{2}(\infty)=\frac{P_{1}+P_{2}}{K_{2}}+\frac{P_{1}+P_{2}+P_{3}}{K_{3}}
\end{gathered}
$$

and

$$
T_{3}(\infty)=\frac{P_{1}+P_{2}+P_{3}}{K_{3}}
$$

In the electrical calibration run, the power $P_{1}$ is supplied to the core alone $\left(P_{2}=P_{3}=0\right)$, the heat capacities of the core and jacket are equal $\left(C_{1}=C_{2}\right)$, and the heat transfer coefficients are determined according to the aforementioned increases in the asymptotic temperature:

$$
\begin{aligned}
& K_{1}=\frac{P_{1}}{T_{1}^{c}(\infty)-T_{2}^{c}(\infty)}, \\
& K_{2}=\frac{P_{1}}{T_{2}^{c}(\infty)-T_{3}^{c}(\infty)},
\end{aligned}
$$

and

$$
K_{3}=\frac{P_{1}}{T_{3}^{c}(\infty)}
$$

During a run of duration $\tau$, the energy supplied to the core can be expressed as

$$
E_{C}^{c}=\int_{0}^{\tau} P_{1} d t=C_{1} T_{1}^{c}(\tau)\left[1+F_{C}^{c}\right]
$$

where the heat-loss correction factor is:

$$
F_{C}^{c}=\frac{K_{1} \int_{0}^{\tau}\left(T_{1}^{c}-T_{2}^{c}\right) d t}{C_{1} T_{1}^{c}(\tau)} .
$$


The energy supplied to the core and jacket can be expressed as

$$
E_{C+J}^{c}=\int_{0}^{\tau} P_{1} d t=C_{1}\left[T_{1}^{c}(\tau)+T_{2}^{c}(\tau)\right]\left[1+F_{C+J}^{c}\right],
$$

where the corresponding heat-loss correction factor is:

$$
F_{C+J}^{c}=\frac{K_{2} \int_{0}^{\tau}\left(T_{2}^{c}-T_{3}^{c}\right) d t}{C_{1}\left[T_{1}^{c}(\tau)+T_{2}^{c}(\tau)\right]}
$$

The heat capacity of the core component can be estimated using eqn. (16) as follows:

$$
C_{1}=\frac{\int_{0}^{\tau} P_{1} d t-K_{2} \int_{0}^{\tau}\left(T_{2}^{c}-T_{3}^{c}\right) d t}{T_{1}^{c}(\tau)+T_{2}^{c}(\tau)} .
$$

The heat capacity according to eqn. (18) includes the effect of the heat loss to the shield.

In the irradiation run, the power is delivered uniformly to all three bodies, and the energy supplied to the core is estimated by measuring the temperature increase of the core. For an irradiation run of duration $\tau^{\prime}$, the radiation energy supplied to the core can be expressed in terms of the calibration energy of the core and jacket as follows:

$$
E_{C}^{r}=E_{C+J}^{c}\left[\frac{T_{1}^{r}\left(\tau^{\prime}\right)}{T_{1}^{c}(\tau)+T_{2}^{c}(\tau)}\right]\left[\frac{1+F_{C}^{r}}{1+F_{C+J}^{c}}\right],
$$

where the heat-loss compensated value of the heat capacity of the core in eqn. (18) is used when $E_{C+J}^{c}, F_{C+J}^{c}$, and $F_{C}^{r}$ are calculated.

\section{RESULTS AND DISCUSSION}

Simulations for the heat-loss compensated calorimeter model were performed at an initial room temperature of $298 \mathrm{~K}$ in the electrical calibration and irradiation runs. Figure 3(a) shows temperature-time curves for a process involving $100 \mathrm{~s}$ of heating and $400 \mathrm{~s}$ of cooling in the electrical calibration run. During the heating in this run, an electric voltage of $0.5 \mathrm{~V}$ is applied between the ends of the wire, and thus the power supplied to the heater of the core is estimated as

$$
P_{1}=60.5 \mu \mathrm{W}
$$

The temperatures in the plot represent the temperature increases of the core, jacket, and shield above the initial temperature of $298 \mathrm{~K}$. In our simulations, three thermistors for the temperature measurements were assumed to be located at the middle of each body, even though these are not included in the simulation model. Figure 3(b) shows the temperaturetime curves for the process involving $100 \mathrm{~s}$ of heating and $400 \mathrm{~s}$ of cooling in the radiation run. During the heating in this run, a uniform energy is delivered to all over the calorimeter and absorbed into the graphite core, jacket, and shield with a power of $100 \mathrm{~W} / \mathrm{m}^{3}$ and into the 

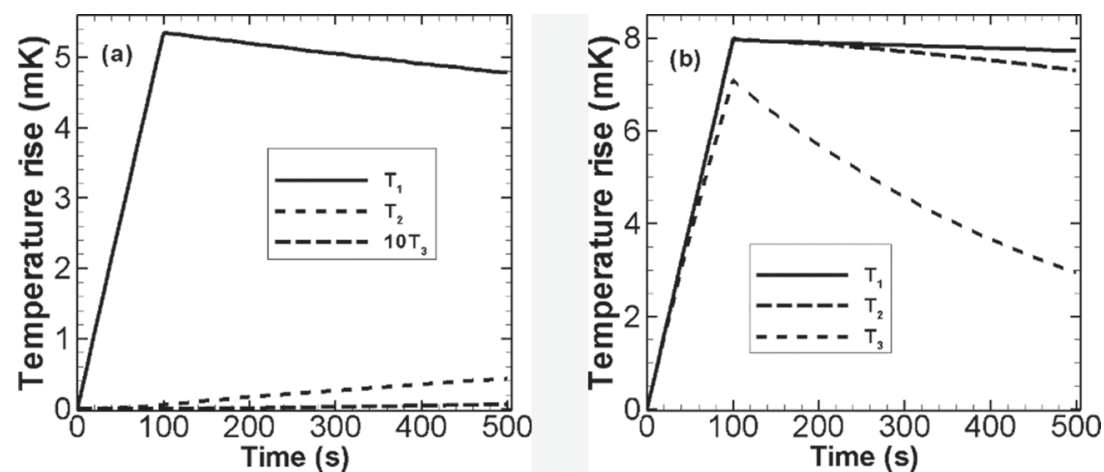

Figure 3: (a) Plot of temperature-time curves for the process of $100 \mathrm{~s}$ of heating and $400 \mathrm{~s}$ of cooling in the electrical calibration run. During the heating in this run, an electric voltage of $0.5 \mathrm{~V}$ was applied between the ends of wire and the power supplied to the heater in the core was $60.5 \mu \mathrm{W}$. (b) Plot of temperature-time curves for the process of $100 \mathrm{~s}$ of heating and $400 \mathrm{~s}$ of cooling in the irradiation run. In the irradiation run, a uniform energy was applied to all the materials and absorbed into the graphite core, jacket, and shield bodies with a value of $100 \mathrm{~W} / \mathrm{m}^{3}$ and into the copper wire with a value of $79 \mathrm{~W} / \mathrm{m}^{3}$.

copper wire with a power of $79 \mathrm{~W} / \mathrm{m}^{3}$. Temperature behaviors in both the calibration and the radiation runs are similar to previous results [1]. The simulations provide details regarding the dissipation energy in each body in the calorimeter model. An energy of $1.26 \mu \mathrm{W}(2.1 \%$ of the input power $60.5 \mu \mathrm{W})$ is dissipated by the radiative heat transfer, and $0.40 \mu \mathrm{W}(0.7 \%)$ is dissipated by the conductive heat leakage through the wire at the elapsed time of $100 \mathrm{~s}$ in the electrical calibration run. At the same elapsed time in the radiation run, an energy of $0.01 \mu \mathrm{W}(0.01 \%$ of the input power $88.2 \mu \mathrm{W})$ is dissipated from the core to the jacket by the radiative heat transfer, and $0.60 \mu \mathrm{W}(0.6 \%)$ by the conductive heat leakage. The amount of the radiative heat transfer from the jacket and the shield at that time is $0.12 \mu \mathrm{W}(0.2 \%$ of the input power $60.5 \mu \mathrm{W})$ in the electrical calibration run and $1.31 \mu \mathrm{W}(1.5 \%$ of the input power $88.2 \mu \mathrm{W}$ ) in the radiation run.

In the electrical calibration run, a constant power of $60.5 \mu \mathrm{W}$ is supplied to the heater for $100,000 \mathrm{~s}$, and the increase in the asymptotic temperature for the core, jacket, and shield are obtained as

$$
\begin{aligned}
& T_{1}^{c}(\infty)=2.40 \times 10^{-1} \mathrm{~K}, \\
& T_{2}^{c}(\infty)=7.10 \times 10^{-2} \mathrm{~K},
\end{aligned}
$$

and

$$
T_{3}^{c}(\infty)=3.00 \times 10^{-3} \mathrm{~K},
$$

respectively, as shown in Fig. 4. The heat transfer coefficients from eqns. (11), (12), and (13) are then determined as

$$
K_{1}=3.58 \times 10^{-4} \mathrm{~W} / \mathrm{K},
$$




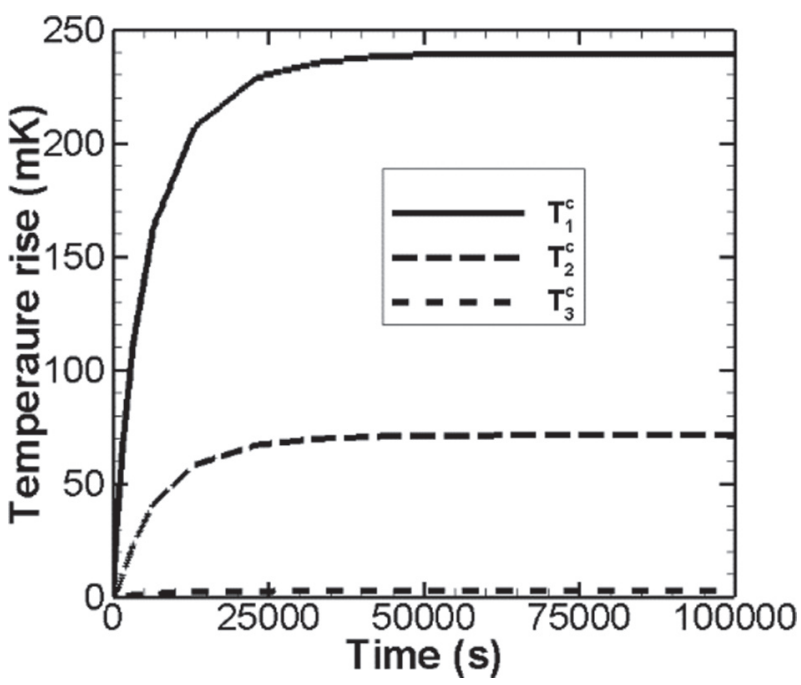

Figure 4: Plot showing the asymptotic temperature increases of the bodies in the calorimeter. A constant power of $60.5 \mu \mathrm{W}$ was supplied to the heater until $100,000 \mathrm{~s}$ in the electrical calibration run.

$$
K_{2}=8.90 \times 10^{-4} \mathrm{~W} / \mathrm{K},
$$

and

$$
K_{3}=2.02 \times 10^{-2} \mathrm{~W} / \mathrm{K} \text {. }
$$

In an electrical calibration run for the time interval of $100 \mathrm{~s}$, the temperature increases of each body are calculated with respect to time. The heat capacity of the core (and jacket) according to eqn. (18) is estimated as

$$
C_{1}=C_{2}=1.118 \mathrm{~J} / \mathrm{K}
$$

which is compared with the actual value obtained from the volume integration in the simulation model i.e. $1.106 \mathrm{~J} / \mathrm{K}$. The difference of $1.1 \%$ between the two values may be due to the heat losses from the core through the conducting wire connected to the heater and the radiative heat transfer to the jacket. In the radiation run, a uniform energy is delivered to all parts of the calorimeter and absorbed into the graphite core, jacket, and shield with a power of $100 \mathrm{~W} / \mathrm{m}^{3}$ and into the copper wire with a power of $79 \mathrm{~W} / \mathrm{m}^{3}$. According to eqn. (19), the radiation energy absorbed into the core during irradiation of $100 \mathrm{~s}$ is estimated as $E_{C}^{r}=8.89 \times 10^{-3} \mathrm{~J}$. This values has a difference of $0.8 \%$ compared with the input energy, $8.82 \times 10^{-3} \mathrm{~J}$, obtained from the input power.

To investigate the effect of the change of the input power in the electrical calibration run on the estimation of the radiation energy absorbed into the core during irradiation, another electrical calibration run with an input power of $P_{1}=118.6 \mu \mathrm{W}$ was performed by increasing the electrical voltage to $0.7 \mathrm{~V}$, yielding $C_{1}=1.117 \mathrm{~J} / \mathrm{K}$ and $E_{C}^{r}=8.88 \times 10^{-3} \mathrm{~J}$. The results show that the change of the input power in the electrical calibration run does not significantly affect the estimation of the absorbed energy of the core. 
The heat loss due to the wire conduction in the three-body graphite calorimeter is reported to be $0.6-0.7 \%$ of the input power supplied to the core [15]. The effect of this conductive heat loss of the wire connected to the heater at the center the calorimeter is investigated by comparing the differences in the estimations of the energy absorbed to the core between the cases with and without the heat loss to the wire. The heat loss to the wire can be removed by simply setting the thermal conductivity of the wire as zero. After the electrical calibration and irradiation runs are repeated without heat loss to the wire, the energy absorbed to the core during the irradiation has a difference of $0.09 \%$, which is one tenth of the estimated value for the case with heat loss to the wire, though an effect of the wire length wire would remain a question. Actually, the heat loss due to the wire conduction of the thermistor for measuring the temperature in each body should also have been estimated.

Figure 5 shows a plot of the temperature profile along the radial direction inside the core in the electrical calibration run. The temperature shows a sharp declination at the center where the heater is located. Such a temperature gradient in the core might affect the estimation of the energy absorbed into the core because the measurement of the temperature using the sensor thermistor depends on the position of the sensor in the core. In our model, the core temperature is calculated at the three different positions of the core thermistor without modeling it, that is, very close to the heater wire at the center, at the middle of the right-half core, and at the rim of the core with the $100 \mathrm{~s}$ of heating in the electrical calibration run, and in the ensuing radiation run the energies absorbed into the core for the three positions of the thermistor are estimated, respectively. The differences of the energy absorbed into the core are $0.96 \%, 0.78 \%$, and $0.94 \%$ at the center, middle, rim positions of the thermistor, respectively. In the calculations, the heat loss due to the wire conduction of the thermistor was not included. The estimated differences are not significant, even if the temperatures measured at the different positions are noticeably different. These results might, however, lack credibility because the axial-symmetry model does not allow the point-typed thermistor to be located at

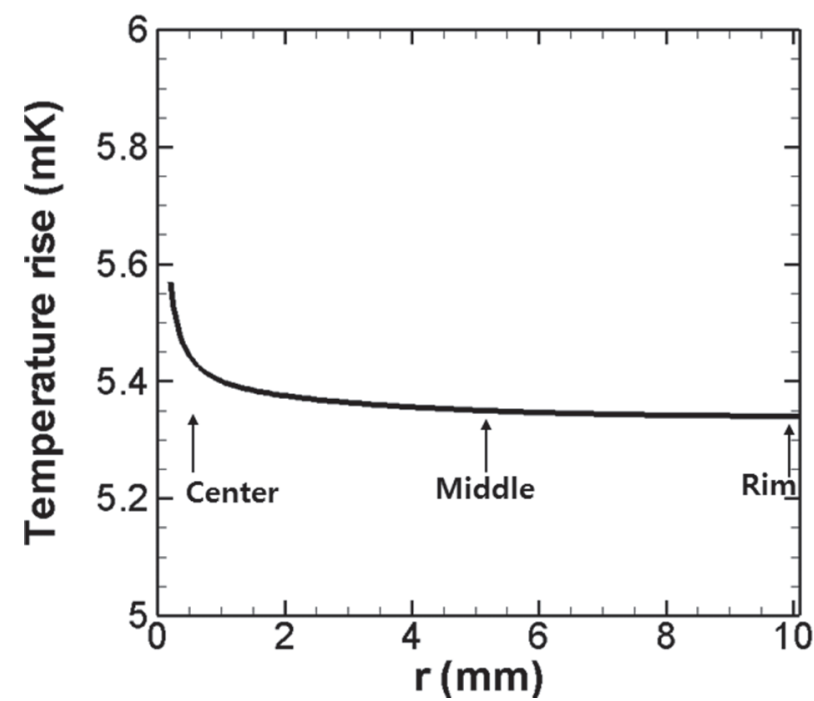

Figure 5: Plot of the temperature profile along the radial direction inside the core at $100 \mathrm{~s}$ in the electrical calibration run. 
off-centers. In the future we need to expand to three-dimensional calorimeter model to probe the restrictions and limits, which the two-dimensional axial-symmetric model would involve.

\section{CONCLUSIONS}

Simulations for a heat-loss compensated calorimeter model were performed using the finite element method in electrical calibration and irradiation runs. The temperature drifts and the heat dissipation of the bodies via conduction and radiative transfer during electrical heating were considered. The simulation was applied to the heating process during the electrical calibration and irradiation. According to the simulation results, dissipation energies from the core by the radiative heat transfer to the jacket and the conductive heat leakage through the wire, respectively, were $2.1 \%$ and $0.7 \%$ of the input power at an elapsed time in the electrical calibration run, while they were $0.01 \%$ and $0.6 \%$, respectively, at the same elapsed time in the radiation run. Our results show that the radiation energy absorbed into the core during irradiation was estimated within an error of $0.8 \%$ using the heat-loss compensated model. The results of additional simulations to determine the effects of the wire conduction of the thermistor on the heat loss suggest that a correction factor for the heat loss due to the wire conduction should be included in the estimation of the absorbed dose during irradiation. In the future, it will be interesting to expand to the three-dimensional calorimeter model for a probe into the restrictions and limits which the two-dimensional axial-symmetric model might involve.

\section{REFERENCES}

[1] Domen, S.R. \& Lamperti, P.J., A heat-loss-compensated calorimeter: theory, design, and performance. Journal of Research of the National Bureau of Standards Section A: Physics and Chemistry, 78A, pp. 595-610, 1974. https://doi.org/10.6028/jres.078a.037

[2] Witzani, J., Duftschmid, K.E., Strachotinsky, C. \& Leitner A., A graphite absorbed-dose calorimeter in the quasi-isothermal mode of operation. Metrologia, 20(3), pp. 73-79, 1984. https://doi.org/10.1088/0026-1394/20/3/001

[3] Fathi, K., Galer, S., Kirkby, K.J., Palmans H. \& Nisbet A., Coupling Monte Carlo simulation with thermal analysis for correcting microdosimetric spectra from a novel micro-calorimeter. Radiation Physics and Chemistry, 140, pp. 406-411, 2017. https:// doi.org/10.1016/j.radphyschem.2017.02.055

[4] Renaud, J., Sarfehnia, A., Bancheri, J. \& Seuntjens, J., Aerrow: A probe-format graphite calorimeter for absolute dosimetry of high-energy photon beams in the clinical environment. Medical Physics, 45(1), pp. 414-428, 2018. https://doi.org/10.1002/mp.12669

[5] Daures, J. \& Ostrowsky, A., New constant-temperature operating mode for graphite calorimeter at LNE-LNHB. Physics in Medicine and Biology, 50(17), pp. 4035-4052, 2005. https://doi.org/10.1088/0031-9155/50/17/008

[6] Janssens, A., Cottens E., Paulsen, A. \& Poffijn, A., Equilibration of a graphite absorbeddose calorimeter and the quasi-isothermal mode of operation. Metrologia, 22(4), pp. 265-270, 1986. https://doi.org/10.1088/0026-1394/22/4/005

[7] Cottens, E., Janssens, A., Eggermont, G. \& Jacobs, R., Absorbed Dose Calorimetry with a Graphite Calorimeter, and G-Value Determinations for the Fricke Dose Meter in High-Energy Electron Beams Int. Symp. Biomedical Dosimetry: Physical Aspects, Instrumentation, Calibration (IAEA-SM-249/32), Vienna: IAEA, pp. 189, 1981.

[8] Domen, S.R., Emissivity of aluminized Mylar. International Journal of Radiation Applications and Instrumentation. Part C. Radiation Physics and Chemistry, 37(2), pp. 199-201, 1991. 
[9] https://www.comsol.com/

[10] Cheng, D.K., Field and Wave Electromagnetics, Addison-Wesley Publishing, MA, 1989.

[11] Jin, J., The Finite Element Method in Electromagnetics, John Wiley \& Sons, New York, 1993.

[12] Popovic, B.D., Introductory Engineering Electromagnetics, Addison-Wesley Publishing, MA, 1971.

[13] Incropera, F.P. \& DeWitt, D.P., Fundamentals of Heat and Mass Transfer, 4th ed., John Wiley \& Sons, New York, 1996.

[14] Cameron, A.D., Casey, J.A. \& Simpson, G.B., NAFEMS Benchmark Tests for Thermal Analysis (Summary), NAFEMS Ltd, Glasgow, 1986.

[15] Radu, D., Guerra, A.S., Ionita, C. \& Astefanoaei, I., Heat loss through connecting thermistor wires in a three-body graphite calorimeter. Metrologia, 47(3), pp. 179-191, 2010. https://doi.org/10.1088/0026-1394/47/3/009 